\title{
The Significance of WiMax on Resource Sharing among Saudi Universities: A Study of King Khalid University, Kingdom of Saudi Arabia
}

\author{
Saeed Q. Y. Al-Khalidi Al-Maliki
}

\begin{abstract}
WiMAX, the Worldwide Interoperability for Microwave Access is a new technology designed for sharing all kinds of data over long distances using wireless communication. It can be used to cover an area of a few square kilometres, such as a college campus or a small town. Its reach is greater than the more familiar "Wi-Fi" networks often employed in offices, homes or coffee shops. Sharing resources such as digital books and articles,E-learning materials, and E-services is necessary for the entire user in frequent and uninterrupted access.This study uses a sample approach to enhance the sharing of digital library resources including medical data, books, and other administrative data resources between universities and other entities. It is proposed that WiMAX technology should be implemented for sharing digital library resources among the various colleges within King Khalid University (KKU), Saudi Arabia. Successful implementation of this model will justify extending it to other universities.
\end{abstract}

Index Terms-WiMAX, digital library, base station, mobile station, backhaul.

\section{INTRODUCTION}

WiMAX is a wide area wireless network standard. IEEE 802.16 has the capability to provide high speed Internet access to rural and urban locations not within range of standard wired networks. WiMAX provides high speed connections of up to $70 \mathrm{Mbps}$ over an area of twenty to thirty square miles. It is also an alternative to satellite Internet services, and is sometimes called wireless MAN [1], [2]. WiMAX differs in performance speed and area covariance, and is increasingly deployed for research because of the service they provide is more efficient than other wireless models. However, WiMAX is a new technology not yet widely deployed because of security threats, so security is the main issue for WiMAX technology. There are two main points at which WiMAX networks are vulnerable to attacks: the subscriber station (SS) and the base station (BS).

There are many different types of attack to which wireless and WiMAX networks may be subject, including repudiation, fabrication, modification, and interception attacks. Here, however, our focus is on constructing an efficient WiMAX connection for the educational users [1], [3]. There are various kinds of threats for WiMAX such as the base station attack, which is when the attacker station imitates a legitimate base station. This may occur due to inadequate two-way

Manuscript received June 6, 2014; revised August 12, 2014.

Saeed Q. Y. Al-Khalidi Al-Maliki is with the Department of Managemen Information Systems, College of Administrative and Financial Sciences, King Khalid University (KKU), KSA (e-mail: salkhalidi@kku.edu.sa). authentication protocols between the base station and subscriber station or if there is no verification of the base station [1], [4]. To avoid these problems we will try to determine the drawbacks of the existing security structure for WiMAX before then discussing these drawbacks briefly and, finally, proposing a new model for ways in which WiMAX networks can rectify the problem.

With millions of dollars invested, digital libraries have emerged as an increasingly important web-based application worldwide [5]. Interest in the research and development of digital libraries has grown rapidly, especially with the appearance of special journal issues, conferences and workshops, and new print and online journals [6]. For the purpose of this study, digital libraries can be thought of as electronic collections that are much richer in content and more capable in functionality than databases or information retrieval systems [6], with data accessibility over the Internet an important ingredient [7].

The major advantages of digital libraries over paper libraries include the fact that resources stored in digital form are easier to manage, the fact that it is possible to provide remote, fast, and fair access to digital library collections, and the way in which digital search techniques offer increased flexibility and power to users [8]. Existing digital library research has focused on the technical development of such systems, such as information storage, information retrieval, and system integration [9], [10]. Most of the prior research is thus comprised of in-depth case studies of individual digital libraries [11].

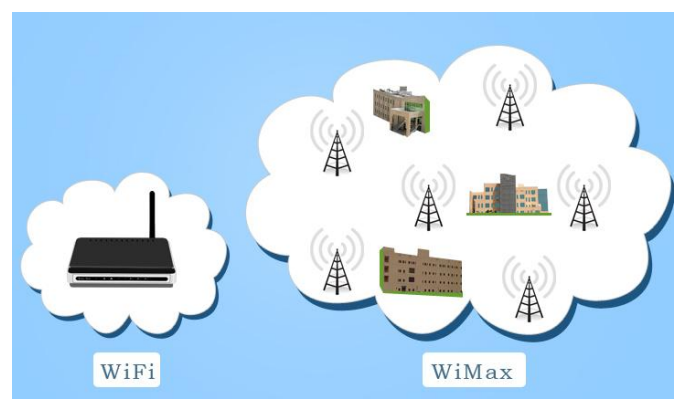

Fig. 1. Accessibility of Wi-Fi (left) and WiMAX (right).

In Fig. 1 we will examine the problems involved in implementing WiMAX and the potential effects of implementation on the use of digital library resources.

\section{BACKGROUND LITERATURE}

ShahidHussain, Muhammad Naeem Khan, Muhammad Ibrahim, in an article entitled "A Security Architecture for 
WiMAX Networks" (2012), proposed a new security model for the WiMax structure. Also, this research article discussed the WiMAX standard, citing the services and long range accessibility etc. In addition to the security issues faced by WiMAX, including threats like message collision attacks, rogue base station attacks, reply attacks, and so on. The proposed WiMAX uses different security mechanisms for unauthorised access, encryption, authentication etc., and several security architecture mechanisms are also implemented. They try to enhance the existing model with the help of the two-way authentication model [1].

In addition, C. L. Borgman [6] explores reasons for the development of digital libraries and the influence of key players within this, while also speculating on the future potential of digital libraries. The author conceives digital libraries as content collected on behalf of user communities, while practising librarians view digital libraries as institutions or services. Tension exists between these communities over the scope and concept of the term "library". Research-oriented definitions serve to build a community of researchers and to focus attention on problems which need to be addressed [6].

Moreover, Zahangir Alam, Colin Patra, Charles Chittaranjan Patra, and M. Abdus Sobhan [12] discussed implementinga new technique to enable all people to use ICT at a very low cost by using smartphone technology for e-education. They try to explore the deployment strategy of the Worldwide Interoperability of Microwave Access network with integration of smartphone technology to interconnect educational organizations with WiMAX broadband Internet connection. They suggest E-learning by smartphone because it provides easy access. The research proposal reduces installation costs and these smart phone techniques will contribute towards a better environmental performance in the educational sector [12].

Also, Paul Pocatilu [13] proposed the implementation of WiMax technology in e-learning centres. His research article discussed the merits and drawbacks of WiMax technology implementation in e-learning centres. The author also suggests implementing the architecture of E-learning centre with the help of strong user authentication, access control, data privacy, data integrity, and sophisticated authentication and encryption technology [13].

Finally, "An overview on WiMAX Security Weaknesses/Potential Solutions" was conducted by Daniel Simion, Mihai-FlorentinUrsuleanu, and Adrian Graur [14] to discuss the existing WiMax Quality of Services and various threats to WiMax technology. The entire research article discussed WiMax security elements and various types of attacks on IEEE 802.16 WiMax technology. The authors are cautious about the use of WiMax technology for public use [14].

\section{RESEARCH SIGNIFICANCE}

King Khalid University is one of the old universities under the control of the Ministry of Higher Education, Saudi Arabia. There are around 70,000 students, making it one of the biggest centres of learning in the Middle East with a standing as a major provider of both further and higher education. It is connected with more than forty colleges for both males and females, as well as a digital library and an e-learning centre. The Deanship of King Khalid University has many resources, such as access to fifty-eight journals, 120,000 Arabic language books, 60,000 English language text books, 600 theses, and 4,000 digital E-books. However, it is necessary to provide a strong communication channel between the colleges, administration, students, and faculty members in order to facilitate the sharing of these resources. Here, we discuss some of the advantages of implementing WiMAX technology to such an end:

\section{A. Coverage and Range}

The maximum cell radius for a Wi-Fi network is an eighth to a quarter of a mile. The range of WiMAX is three to five miles from the antenna, depending on the frequency and topography. This wider coverage means that more customers can be connected to the network at a lower cost.

\section{B. Non-Line-of-Sight Service}

Many 3G mobile phones and radio devices require a line-of-sight (LOS) connection between the access point and the user's modem. The mobile WiMAX standard, however, features 256 point orthogonal frequency-division multiplexing (OFDM) technology, which significantly enhances the NLOS capabilities of the network. This allows the operator to support more customers per cell site due to better signal penetration.

\section{Higher Data Rates}

WiMAX is able to achieve download speeds of up to six Mbps for fixed residential services and up to four Mbps for mobile users. Residential WiMAX is able to achieve a higher data rate because it uses a longer antenna.

\section{Interoperability Advantage}

WiMAX represents a shift in strategy for designing and operating wireless broadband data networks. WiMAX compliant devices from different device manufacturers will be able to operate on the same network. This increases the viability and decreases the risk involved in deploying wireless broadband networks.

\section{E. Improved Quality of Service}

The improved quality of service built into the WiMAX technology platform will enable carriers to offer features designed for use by commercial enterprises. WiMAX will also be a pipeline for media-rich applications for personal use, such as gaming or multicast video (once IPv6 is introduced).

\section{F. Lower Consumer Premises Equipment Cost}

The cost of WiMAX modems and compliant devices will fall as multiple equipment vendors compete with one another to introduce their products. A proprietary system such as Long Term Evaluation (LTE) does not take advantage of economies of scale, or at least not as much as an open standards technology platform.

\section{Proposed ARChitecture Model}

WiMAX technology provides connectivity with desktop computers, laptops, and even mobile devices. For example, 
we can connect to the Internet through our WiMAX connection at work, at the library, at home, at the E-learning centre on the University campus, and even at the seaside, as the WiMAX service provides network coverage to all of these places in KKU in Fig. 2. With the help of this technology, we can even provide cheap and free Voice over Internet Protocol (VoIP) phone calls for those using a WiMAX enabled mobile phone or a laptop computer.

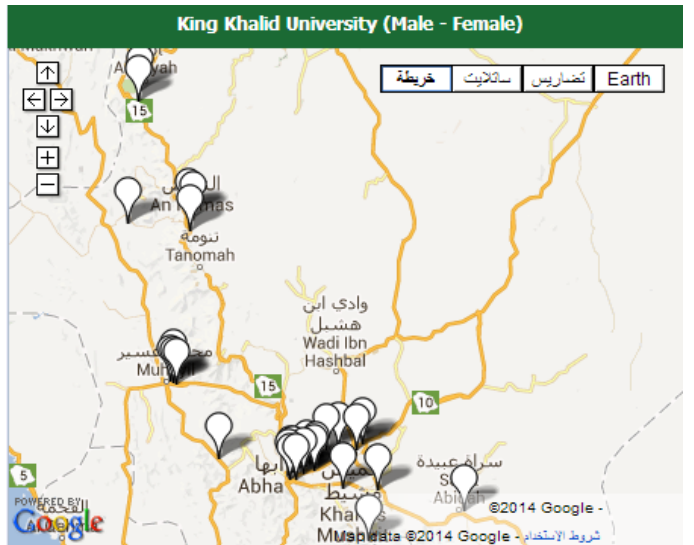

Fig. 2. Locations of colleges affiliated to KKU(Source: Google maps).

The WiMAX Forum NWG network reference model defines three basic architectural entities: the mobile station MS), the access service network (ASN), and the connectivity service network (CSN). The role of the MS is to provide users with access to the WiMAX network. The ASN is the radio access network and is formed by numerous base stations (BSs) and ASN gateways (ASN-GWs), and managed by a network access provider (NAP). CSN is the network entity providing IP connectivity to the WiMAX radio equipment, including all the IP core network functions required for internetworking with the rest of the world. CSNs are maintained by Network Service Providers (NSPs).

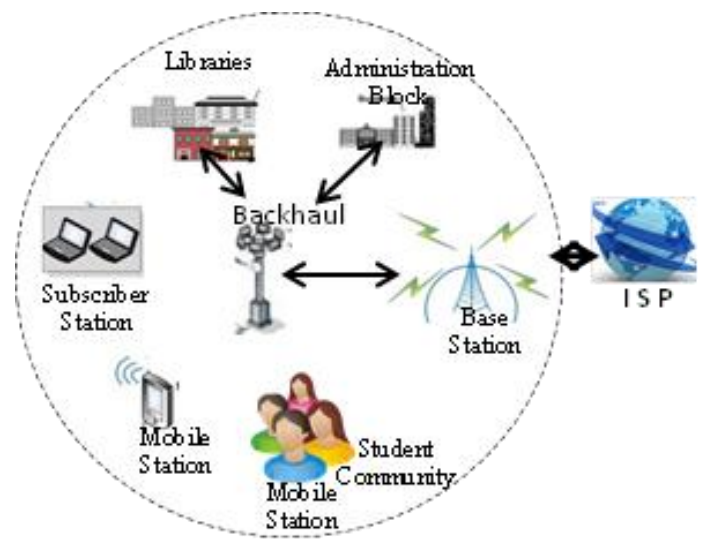

Fig. 3. WiMAX connectivity with libraries.

In Fig. 3 the study will establish a connection to the library system using the administration block at KKU as a base station with backhaul and ASN gateways. If it is necessary, we will use more backhaul to provide an efficient service to the students and faculties, providing access to digital resources from the library. Furthermore, we can also extend our network connectivity to cover mobile stations. This proposed architecture is an attempt to develop an effective network connection. After successful implementation, we will extend these networks to other colleges affiliated with
KKU.

Providing access to the digital library becomes easier in view of the fact that only one place needs to be supervised, not thousands of computers belonging to a university. Also, the security changes can be easily tested and implemented using a unique entry point for all of the stations. Wireless data communication can be easily monitored, so high security needs to be assured by using specific standards.

\section{BENEFITS OF THE PROPOSED MODEL}

WiMAX technology provides up to thirty miles of linear service area range, and WiMAX allows users to obtain broadband connectivity wirelessly. Within a typical cell radius deployment of three to five miles, users do not require line of sight, and systems can be expected to deliver shared throughput of up to seventy Mbps, which is enough bandwidth to simultaneously support sixty businesses with T1-type connectivity and hundreds of residences with DSL-type connectivity.

WiMAX thus delivers a cost-effective alternative to broadband access. Since many businesses and college communities are not zoned for cable, their local telecom companies provide the only option for broadband service. The ease of deployment for WiMAX systems can benefit customers by bringing new competition into the marketplace and lowering prices, or by enabling enterprises and higher-educational institutions to set up their own private networks. Moreover, the added benefit that remote WiMAX users need less or no on-site installation significantly reduces the cost and set-up time of the service, allowing it to compete favourably with existing cable or other wired solutions.

\section{A. Benefits to Students}

- 1,000 students can access the network within a radius of 1.5 miles from a tower, which equates to seven square miles of unlimited data access at university as well as at home.

- Students can interact with each other and with the practitioner instead of hiding behind large monitors.

- It is much easier to accommodate several mobile devices in a classroom than several desktop computers.

- PDAs or tablets holding notes and E-books are lighter and less bulky than bags full of files, papers, and textbooks, or even laptops.

- Handwriting with the stylus pen is more intuitive than using a keyboard and mouse.

- It is possible to share assignments and work collaboratively.

- Mobile devices can be used anywhere, anytime, including at home, on the train, or in hotels; this is invaluable for work-based training.

- WiMAX provides unparalleled digital library access and thus gives more opportunities for students to increase their knowledge.

\section{B. Benefits to Administration}

- Administrative staff can work from home using mobile, VoIP enabled communication devices, and can access useful mobile services. 
- WiMAX provides an easy and quick method of communicating with other colleges.

- Wireless networks require significantly less maintenance than cable-based networks.

- WiMAX technology can be used to support suburban areas.

\section{Benefits to Staff}

- WiMAX ensures unlimited access to digital library resources for teaching and research.

- WiMAX technology also enables mobile communication.

\section{CONCLUSION}

Increasing access to the Internet for resource and data sharing has the potential to make using digital library resources highly desirable among students and teachers alike. The objective of this research is to study the acceptance of WiMAX technology by focusing on higher education students in KKU, Saudi Arabia, and also to examine factors that have a positive influence on behavioural intention to use wireless and broadband communication channels. This study will provide insights into overall improvement of data transmission and wireless communication technology, better bandwidth utilisation, and reduction in computational complexity. Research suggests that a positive attitude leads to the behavioural intention to use WiMAX technology. Therefore, the university administration should focus on designing WiMAX technology that appeals to students. Fostering a positive perception of WiMAX technology and university support are two major factors within the successful implementation of any learning system.

\section{REFERENCES}

[1] S. Hussain, M. N. Khan, and M. Ibrahim, "A security architecture for WiMAX networks," International Journal of Computer Applications, vol. 50, no. 9, pp. 35-39, July 2012.

[2] P. Rengaraju, C. Lung, and A. Srinivasan, "Design of distributed security architecture for multi hop WiMAX networks," presented at
Eighth Annual International Conference on Privacy, Security and Trust, IEEE 2010.

[3] S. P. Ahuja and N. Collier, "An assessment of WiMAX security," Communications and Network, vol. 2, no. 3, pp. 134-137, 2010.

[4] K. D. Deepti and S. P. Ahuja, "A survey of rogue base station attack in WiMAX/IEEE802.16," International Journal of Advanced Research in Computer Science and Software Engineering, vol. 2, no. 1, 2012.

[5] L. F. Lunin and E. A. Fox, "Perspectives on digital libraries: introduction and overview," Journal of the American Society for Information Science, vol. 44, no. 8, pp. 440-445, 1993.

[6] C. L. Borgman, "What are digital libraries? Competing visions," Information Processing and Management, vol. 35, no. 3, pp. 227-243, 1999.

[7] National Science Foundation. (1993). E-learning. Digital Libraries Initiative Phase 2. [Online]. Available: http://www.nsf.gov/

[8] G. Wiederhold, "Digital libraries, value, and productivity," Communications of the ACM, vol. 38, no. 12, pp. 85-90, 1995.

[9] R. Rao, J. O. Pedersen et al., "Rich interaction in the digital library," Communications of the ACM, vol. 38, no. 4, pp. 29-39, 1995.

[10] C. Watters, "Information retrieval and the virtual document," Journal of the American Society for Information Science, vol. 50, no. 11, pp. 1028-1029, 1999.

[11] E. A. Fox, D. Hix et al., "Users, user interfaces, and objects: envision, a digital library," Journal of the American Society for Information Science, vol. 44, no. 8, pp. 480-491, 1993.

[12] Z. Alam, C. Patra et al., "Smart phone based internet connectivity to educational institutions: Context digital Bangladesh," Advances in Mathematical and Computational Methods, vol. 2, no. 4, pp. 33-38, 2012.

[13] P. Pocatilu, "Using WiMAX technology for e-learning solutions," Oeconomics of Knowledge, vol. 2, no. 3, pp. 6-10, 2010.

[14] D. Simion, M.-F. Ursuleanu, and A. Graur, "An Overview on WiMAX Security Weaknesses/Potential Solutions," in Proc. $11^{\text {th }}$ International Conference on Development and Application Systems, Suceava, Romania, pp. 98-102, May 17-19, 2012.

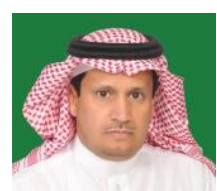

Saeed Q. Y. Al-Khalidi Al-Maliki is the dean of Libraries Affairs at King Khalid University (KKU), Kingdom of Saudi Arabia. He has published many papers in international journals. Dr. Al-Khalidi completed a master degree and a doctorate (Ph.D) in information systems in England. He is a faculty member in the Department of Management Information Systems, College of Administrative and Financial Sciences, King Khalid University (KKU), KSA. His research interests include information system development, approaches to systems analysis and the early stages of the systems development process, IT/IS evaluation practices, and e-readiness assessment. 